\title{
Surgical management of trigeminal schwannomas
}

\author{
Ricardo Ramina, M.D., Ph.D., ${ }^{1,2}$ Tobias A. Mattei, M.D., ${ }^{1}$ Marília G. SóRia, ${ }^{1}$ \\ Erasmo B. da Silva Jr., M.D., ${ }^{1,2}$ André G. Leal, M.D., ${ }^{1,2}$ Maurício C. Neto, M.D., ${ }^{1}$ \\ and Yvens B. Fernandes, M.D., Ph.D. ${ }^{3}$ \\ ${ }^{1}$ Department of Neurosurgery, Instituto de Neurologia de Curitiba; ${ }^{2}$ Postgraduate Course in Surgery, \\ Pontifícia Universidade Católica do Paraná, Curitiba, Paraná; and ${ }^{3}$ Department of Neurosurgery, \\ Universidade Estadual de Campinas, São Paulo, Brazil
}

\begin{abstract}
Object. The authors provide a detailed review of the surgical management of trigeminal schwannomas (TSs) and also discuss the best surgical approach based on the surgical anatomy and tumor extension.

Methods. A series of 17 patients with TSs who were surgically treated between 1987 and 2008 at the authors' institution is reported. The lesions were small $(<3 \mathrm{~cm})$ in 2 , medium (between 3 and $4 \mathrm{~cm})$ in 5 , large $(>4 \mathrm{~cm})$ in 6 , and giant $(>5 \mathrm{~cm})$ in 4 cases. Preoperative symptoms included trigeminal hypesthesia (53\%), facial pain (53\%), headaches $(35.3 \%)$, hearing impairment $(17.6 \%)$, seizures $(17.6 \%)$, diplopia $(11.8 \%)$, ataxia $(11.8 \%)$, and hemiparesis and increased intracranial pressure with papilledema (5.9\%). The mean follow-up duration was 10.5 years (121.6 months), with an average of 0.8 patients per year.

Results. Total tumor excision was possible in 16 patients, with no surgery-related deaths. Postoperative trigeminal anesthesia was observed in 7; trigeminal motor function was preserved in 7 . Two developed cerebrospinal fluid leakage, 2 presented with mild facial palsy, and 1 patient with neurofibromatosis Type 2 had recurrence of the tumor, which was uneventfully removed. Of the 9 who reported facial pain, only 1 remained symptomatic postoperatively.

Conclusions. The best treatment for TSs is complete microsurgical removal. Postoperative preservation of trigeminal nerve function is possible when resection of the lesion is performed at well-established skull base neurosurgical centers. Although good results have been reported with radiosurgery, no cure can be obtained with this therapeutic modality. Instead, this treatment should be reserved only for nonresectable and residual tumors within the cavernous sinus. (DOI: 10.3171/FOC.2008.25.12.E6)
\end{abstract}

\section{KEY WORDS • disease classification • neurosurgical technique • trigeminal schwannoma}

$\mathrm{S}$ CHWANNOMAS originating from cranial nerves are usually benign, isolated, and slow growing. They may occur in multiple sites when associated with NF2. Schwannomas arising from the trigeminal nerves are the second most common type of intracranial schwannomas, representing $0.8-8 \%$ of these tumors..$^{5,29,38,46}$ Trigeminal schwannomas tend to occur in middle-aged patients; the highest incidences are between the ages of 38 and 40 years, and are more common in women. ${ }^{23,24,45} \mathrm{~Pa}-$ tients with TS frequently complain of trigeminal nerverelated symptoms, but they may also be asymptomatic. In a recent review of the literature, Samii et al. ${ }^{41}$ reported trigeminal nerve symptoms in $51 \%$ of patients, followed by headaches $(16 \%)$ and diplopia $(11 \%)$. The treatment of choice remains resection. In 1918, Frazier ${ }^{14}$ reported the first surgical removal of a TS. Prior to the advent of CT and MR imaging, the diagnosis of TS was difficult.

\footnotetext{
Abbreviations used in this paper: $\mathrm{CSF}=$ cerebrospinal fluid; $\mathrm{ICP}=$ intracranial pressure; $\mathrm{NF} 2=$ neurofibromatosis Type $2 ; \mathrm{TS}=$ trigeminal schwannoma.
}

Indeed, high death rates had been associated with surgical treatment prior to the late $1950 \mathrm{~s} .{ }^{43}$ Since then, in addition to a large series of 111 patients, ${ }^{24}$ several small series $^{7,9,27,29,41}$ have demonstrated positive results and low mortality and morbidity rates associated with complete resection. Different surgical approaches may be used depending on the location and extension of the lesion.

\section{Surgical Anatomy}

Recent advances in understanding the microsurgical anatomy of skull base structures are hallmarks of modern neurosurgery. Findings reported in the literature have proven, specifically in trigeminal nerve schwannomas, that a precise 3D knowledge of the dural, venous, and nerve anatomy of the cavernous sinus acquired through dissections of laboratory specimens is mandatory. $9,10,42$ Moreover, clinical surgical experience in resecting tumors within such a complex region is necessary to remove the maximum amount of the tumor safely. ${ }^{12,13}$

The trigeminal nerve emerges from the ventrolateral surface of the pons and runs anteriorly $\sim 1-2 \mathrm{~cm}^{6}$ 
R. Ramina et al.

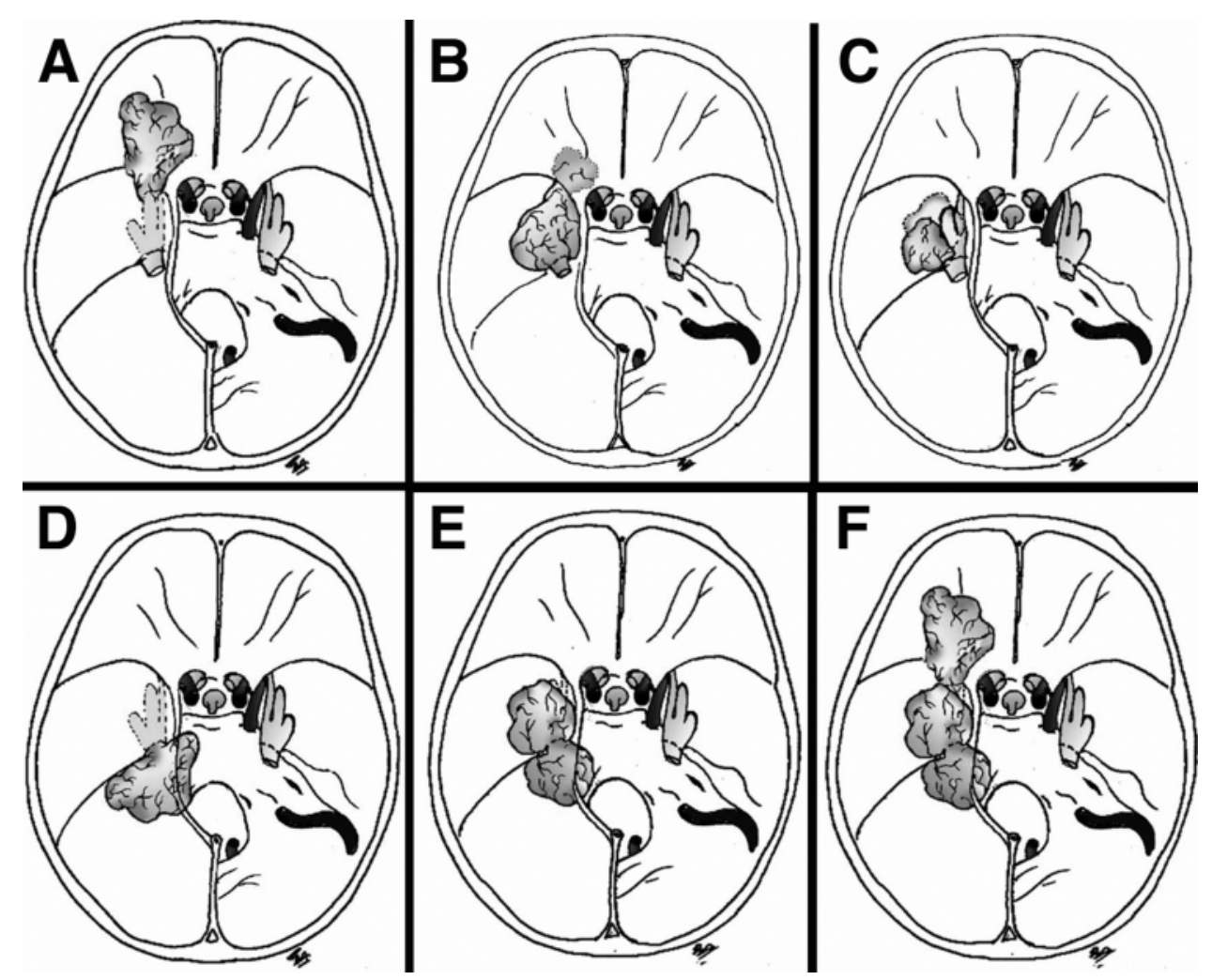

FIG. 1. Drawings showing the 6 types of TS. A: Large extracranial TS with a small middle fossa extension (Type A). B: A TS with its main portion in the middle fossa and a small extracranial extension Type B). C: A middle fossa TS (Type C). D: A posterior fossa TS (Type D). E: A TS with middle and posterior fossa extensions (Type E). F: A TS with extracranial, middle, and posterior fossa extensions (Type F).

through the cerebellopontine cistern to reach the petrous apex. Vascular structures such as the petrosal vein and the superior cerebellar artery lie close to the trigeminal nerve. Over the petrous apex, $\sim 7 \mathrm{~mm}$ of distance from the medial lip of the internal acoustic meatus, ${ }^{26}$ the Gasserian ganglion is enveloped by a dural deflection forming the Meckel cave, laterally to the cavernous sinus and the carotid artery. As it leaves the Meckel cave, the trigeminal nerve is divided into 3 branches: the ophthalmic (V1), maxillary (V2), and mandibular (V3) branches. These 3 nerves run under the middle fossa dura mater and leave the temporal bone through the lateral wall of the cavernous sinus (for V1), foramen rotundum (for V2), and foramen ovale (for V3).

The trigeminal nerve can also be surgically classified into 3 segments: cisternal, from the brainstem to the petrous apex; intracranial-extradural, from the Meckel cave to the foramina; and extracranial (V1, V2, and V3).

Functionally, the trigeminal nerve has 2 portions: the "pars compacta," which constitutes the triangular portion and comprises the primary afferent fibers that are responsible for the special sensibility of the face; and the motor root, which carries the branchiomotor fibers to the muscles of mastication. The motor root runs practically separated from the "pars compacta" but together with the cranial portion of the nerve. At the level of the Meckel cave, it is oriented medially and leaves the skull together with the maxillary nerve.
The intracranial-extradural portions of V2 and V3 are surgically identified using the foramen spinosum as an anatomical landmark, which is located at the sphenoid bone and contains the middle meningeal artery. The foramina ovale and rotundum are located $\sim 2-5 \mathrm{~mm}$ superoanteriorly and $10-12 \mathrm{~mm}$ superomedially to the foramen spinosum, respectively.

\section{Classification of Tumor Extension}

Trigeminal schwannomas may originate from the root, the ganglion, or the peripheral branches of the trigeminal nerve. Jefferson ${ }^{22}$ initially divided these tumors into 4 groups depending on their anatomical location: posterior fossa (root type), combined posterior fossa-middle fossa (dumbbell type), middle fossa (ganglion type), and peripheral (division type). Samii et al. ${ }^{41}$ classified the tumor extension into 4 categories based on radiological findings: Type A, intracranial tumor predominantly in the middle fossa; Type $\mathrm{B}$, intracranial tumor predominantly in the posterior fossa; Type $\mathrm{C}$, intracranial dumbbell-shaped tumor in the middle and posterior fossa; and Type D, extracranial tumor with intracranial extensions.

In our institution, we prefer to use the classification of Samii et al. ${ }^{41}$, with the following modifications: Type A, predominantly an extracranial tumor with small extension in the middle fossa (Fig. 1A); Type B, an intracranial tumor predominantly in the middle fossa with extracranial extension (Fig. 1B); Type C, a tumor in the 

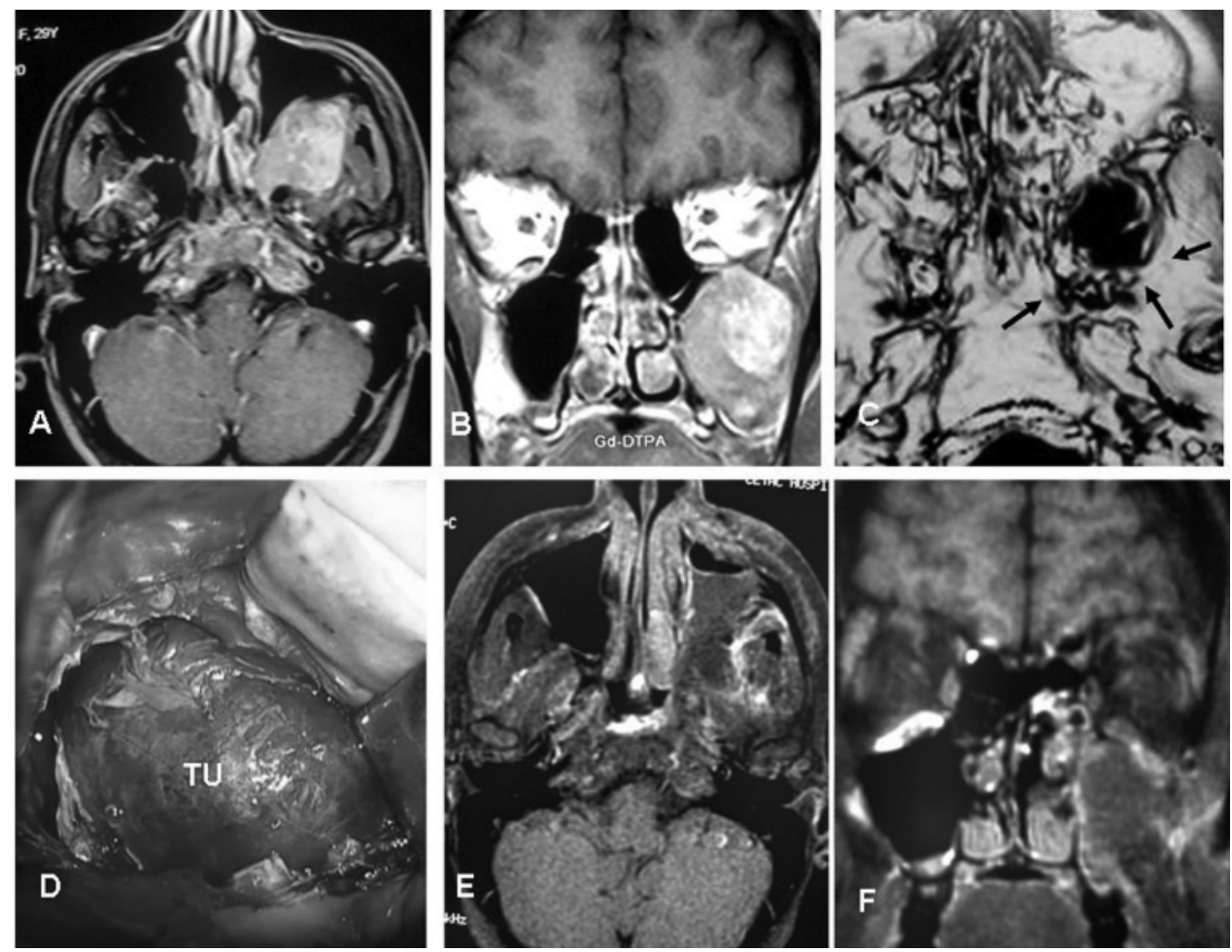

FIG. 2. A and B: Axial and coronal MR imaging studies of a large TS with its main portion in the maxillary sinus (Type A). Gadopentetate was used as the contrast agent. C: A CT scan showing enlargement of the foramen rotundum (arrows). D: Intraoperative photograph showing the transmaxillary approach exposing the tumor (TU). E and F: Postoperative MR images.

middle fossa (Fig. 1C); Type D, a tumor in the posterior fossa (Fig. 1D); Type E, a tumor with middle and posterior fossa extensions (Fig. 1E); and Type F, a tumor with extracranial, middle, and posterior fossa extensions (Fig. $1 F)$. In our opinion, these modifications are important to assure the best surgical approach for each type of lesion. In the experience of the senior author (R.R.), each subgroup of this modified classification presents a different surgical challenge. Tumor types are ordered according to the level of difficulty as A, C, D, B, E, and F. The degree of difficulty in radical surgical removal depends on the particularities of each case, but according to this modified classification, Type A tumors have the lowest level of difficulty and Type F tumors, on the contrary, the highest.

\section{Surgical Approaches}

According to the aforementioned classification (Table 1), Type A tumors are resected via an extradural approach (transmaxillary approach) (Fig. 2) or TM associated with an extradural middle fossa approach if there is broad extension to the middle fossa. Type B lesions are removed through an intradural middle fossa approach; large extradural tumor extensions, however, may require a transmaxillary exposure (Fig. 3). Type $\mathrm{C}$ tumors are extirpated via a middle fossa approach, either intradurally (for large lesions) or extradurally (for small lesions) (Fig. 4). Resection of Type D tumors is best achieved via the retrosigmoid approach (Fig. 5). Type E tumors are extirpated using a presigmoid approach or 2-staged retrosigmoid and middle fossa approaches ${ }^{4}$ (Fig. 6). As stated before, Type
F tumors are considered the most challenging lesions and may demand a combination of all previously described approaches (Fig. 7).

Transmaxillary Approach (Caldwell-Luc Procedure). This procedure is usually performed in association with the middle fossa approach to remove large TSs. In our series, it was used to remove a large schwannoma within

TABLE 1: Types of tumors and surgical approaches in 17 patients with TSs*

\begin{tabular}{lccl}
\hline $\begin{array}{l}\text { Level of } \\
\text { Difficulty }\end{array}$ & $\begin{array}{c}\text { Type of } \\
\text { Tumor }\end{array}$ & $\begin{array}{c}\text { No. of Patients } \\
(\%)\end{array}$ & \multicolumn{1}{c}{ Approach } \\
\hline easiest & A & $2(11.8)$ & $\begin{array}{l}\text { extradural (TM or TM + EMF) } \\
\text { intradural (IMF) }\end{array}$ \\
& B & $2(11.8)$ & \\
& C & $5(29.3)$ & IMF or EMF \\
& D & $2(11.8)$ & $\begin{array}{l}\text { retrosigmoid } \\
\text { presigmoid or 2-staged retrosig- } \\
\text { moid/middle fossa } \\
\end{array}$ \\
& E & $4(23.5)$ & combination of all of the above \\
\hline
\end{tabular}

* EMF = extradural middle fossa; IMF = intradural middle fossa; TM = transmaxillary. 


\section{R. Ramina et al.}

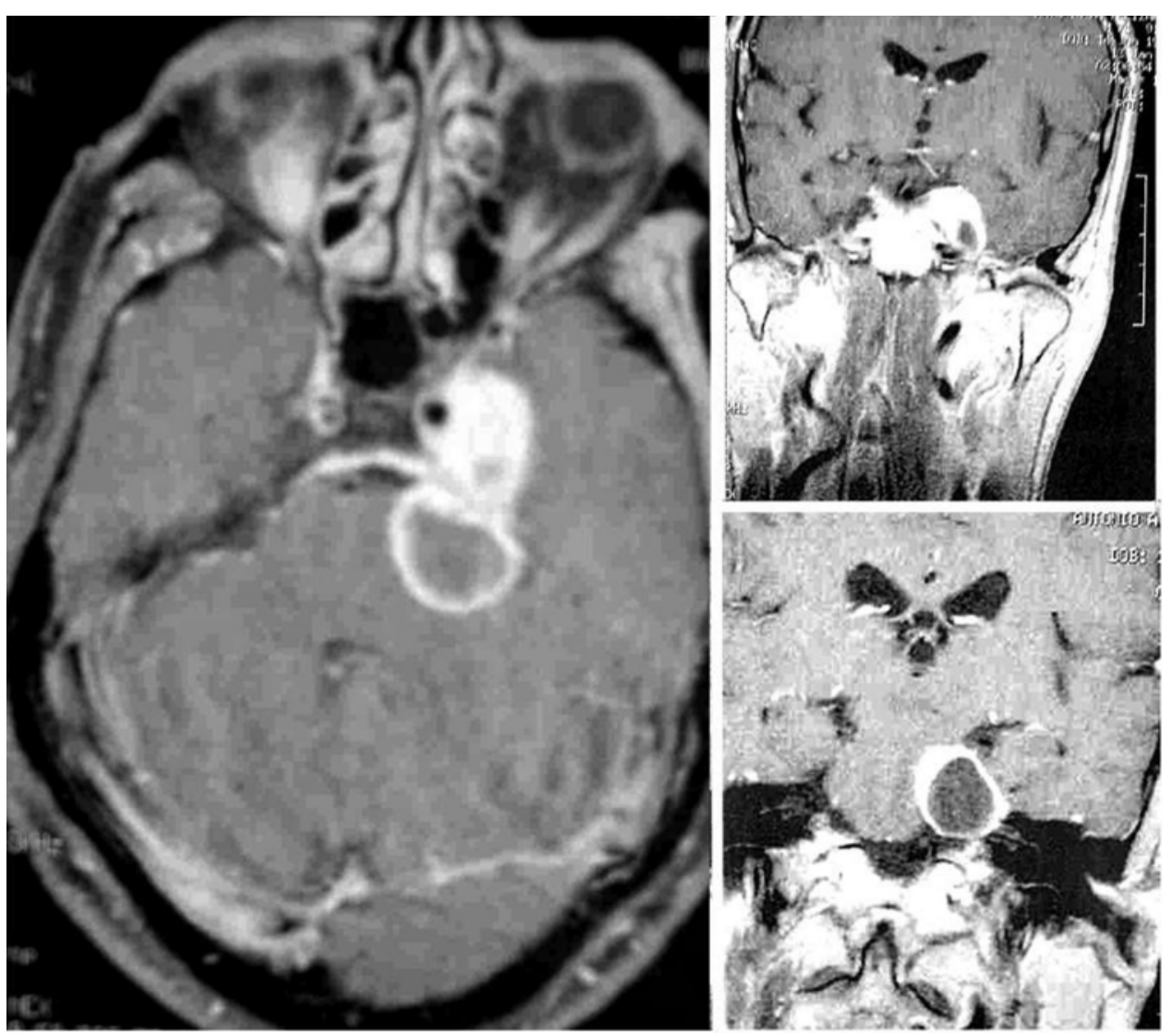

FIG. 4. Preoperative MR imaging studies showing a middle fossa TS with a cystic posterior fossa portion (Type C).

the maxillary sinus with small extension to the middle fossa and the cavernous sinus. After induction of general anesthesia, the gingiva is infiltrated with lidocaine $1 \%$ with 1:100,000 epinephrine solution. A 3-cm incision is made over the upper canine and the upper first premolar tooth. To improve the suturing, $1 \mathrm{~cm}$ of gingival tissue is left intact above the dentition. Soft tissue and periosteum are elevated from the anterior wall of the maxilla. The infraorbital nerve is identified as it exits the infraorbital foramen (midpupillary line). The anterior wall of maxillary sinus is opened. The tumor is identified and microsurgical intracapsular debulking is performed. Total removal of the maxillary, middle fossa, and cavernous sinus portions is accomplished with this approach. The incision is closed with 4-0 absorbable sutures.

Middle Fossa Approach. The patient is placed supine with the head fixed in a 3-pin device and rotated $\sim 30^{\circ}$ to the opposite side. A C-shaped skin incision over the pterium is performed. The temporalis muscle fascia is incised to protect the frontal branch of the facial nerve, and the scalp-fascia flap is lifted anteriorly. The temporalis muscle is retracted inferiorly and posteriorly. A pterional craniotomy is performed and enlarged to the base of the temporal fossa by removing bone with a rongeur to obtain a flat viewing angle across the floor of the middle fossa. The dura mater is then dissected and elevated from the middle fossa, exposing the superior orbital fissure. The middle meningeal artery is coagulated and transected.
The second and third branches of the trigeminal nerve are identified. The foramen ovale and rotundum are exposed using a drill. The dura mater is elevated from the lateral cavernous sinus wall, revealing the tumor extradurally and the branches of the trigeminal nerve. The tumor is debulked and its capsule is dissected from the intact branches of the trigeminal nerve, which allows radical removal of the lesion. Large tumors are approached intradurally by opening the sylvian fissure and exposing the optic nerve, the internal carotid artery, and the third cranial nerve. The lateral cavernous sinus wall is exposed via a temporopolar approach and the tumor is removed. The trigeminal fibers are preserved as much as possible. The petrous apex is drilled and the petroclinoidal ligament is cut to expose the posterior fossa portion, allowing complete excision of the remaining tumor.

Retrosigmoid Approach. This approach is performed by placing the patient in the dorsal (mastoid) position with the head turned to the opposite side and the ipsilateral shoulder elevated. A linear incision is placed $4 \mathrm{~cm}$ behind the external auditory canal. The asterium is exposed to determine the junction of the tranverse and sigmoid sinuses. A craniotomy $4 \mathrm{~cm}$ in diameter is performed, with the superior and anterior margins bordering the transverse and sigmoid sinuses, respectively. The dura mater is opened parallel to the sigmoid sinus; CSF is drained from the cerebellomedullary cistern; and CNs VII-XI are identified. The tumor is thereby exposed near the tentorium 

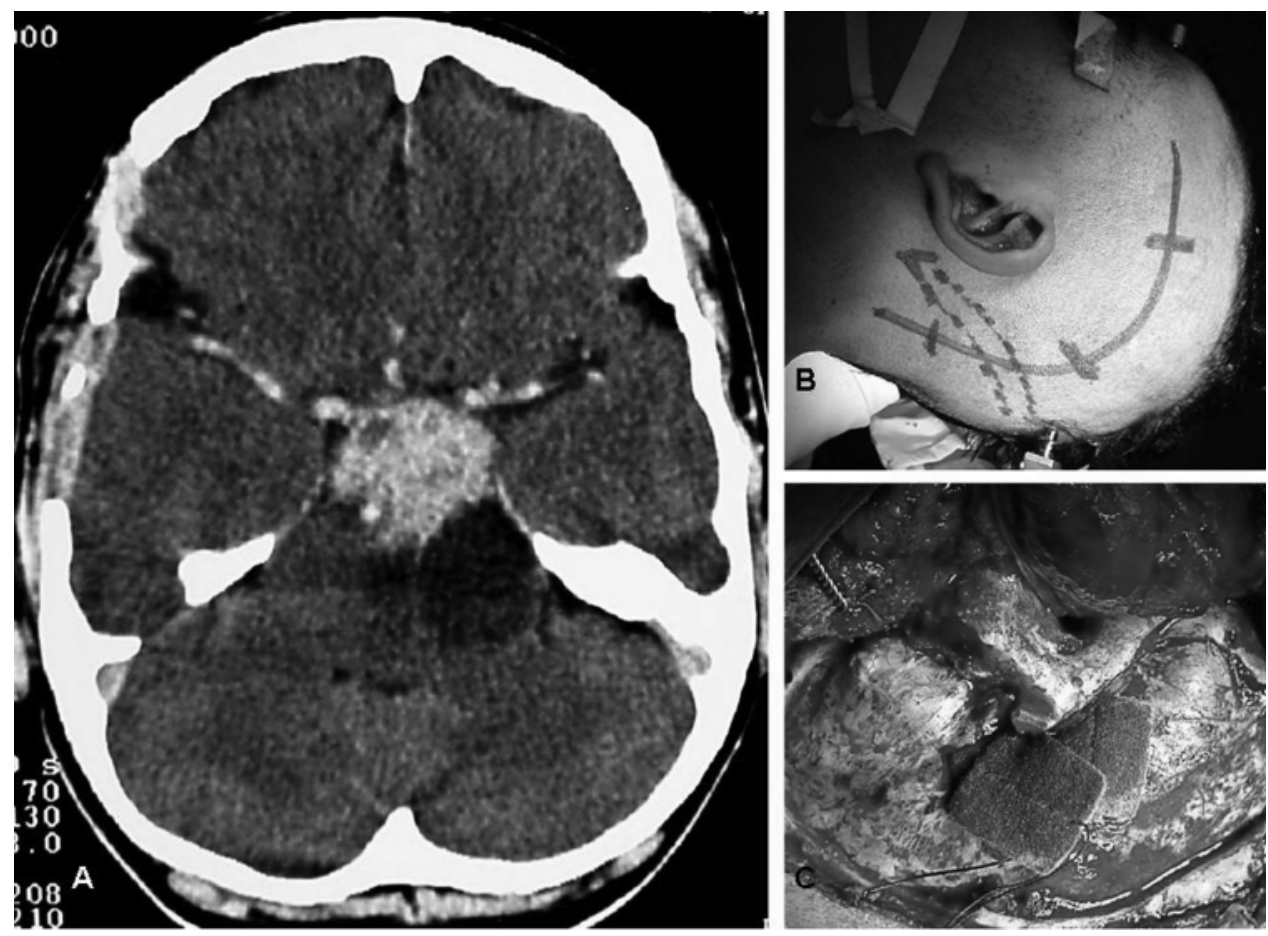

FIG. 5. A: Preoperative MR imaging study showing a posterior fossa TS (Type D) with cystic portion. B: Photograph showing intended path of the skin incision. C: Presigmoid craniotomy.

margin. After intracapsular tumor debulking, microsurgical radical removal is accomplished. A watertight dural closure is completed, and the bone flap is fixed in place. All opened mastoid cells are sealed with bone wax to help prevent CSF leaks.

Presigmoid Approach. The patient is placed in the dorsal (mastoid) position. A C-shaped skin incision from the middle fossa to the mastoid tip is performed. Two bur holes are placed anteriorly and 2 are placed posteriorly at the intersection of the transverse and sigmoid sinuses. The temporal and retromastoid dura mater is exposed through a craniotomy. Mastoidectomy with preservation of the labyrinth block and the facial nerve canal is the next surgical step. Parallel dural incisions to the middle fossa floor are made anteriorly to the sigmoid sinus. The superior petrosal sinus is then ligated and transected. At this point, the inferior temporal lobe and the lateral portion of the cerebellum are slightly retracted so that the vein of Labbé is preserved. The tumor is identified and the seventh and eighth cranial nerves are usually displaced inferiorly. The tumor within the cavernous sinus is removed after opening its lateral wall. After complete excision of the tumor, the dura maer is closed in a watertight fashion and the skull base is reconstructed with myofascial flaps. ${ }^{39}$

\section{Methods}

This series includes 17 patients who were surgically treated between 1987 and 2008 at our institution. Two patients received a diagnosis of NF2. One patient presented with a posterior communicating artery aneurysm on the same side as the TS. There were 2 Type A, 2 Type B, 5 Type C, 2 Type D, 4 Type E, and 2 Type F tumors (Table $1)$. The cavernous sinus was involved in 13 cases. The sizes of the lesions were small $(<3 \mathrm{~cm})$ in 2 , medium (between 3 and $4 \mathrm{~cm}$ ) in 5, large $(>4 \mathrm{~cm})$ in 6 , and giant $(>5 \mathrm{~cm})$ in 4 cases.

The clinical symptoms in these patients included trigeminal hypesthesia (9 patients), facial pain (9 patients), headaches (6 patients), hearing impairment (3 patients), seizures ( 3 patients), diplopia (2 patients), ataxia (2 patients), and hemiparesis and increased ICP with papilledema (1 patient) (Table 2). Prior to 1990, the extent of resection was confirmed by postoperative imaging. After 1990 all patients underwent pre- and postoperative CT and MR imaging studies. In our series, postoperative imaging examinations were routinely performed 3 days after surgery. Recurrence was defined as a new lesion identified on routine MR imaging in a patient considered to have had total resection. Progression was related to tumor growth in a patient considered to have had subtotal or partial resection, as demonstrated by immediate postoperative MR imaging. Postoperatively, imaging examinations are performed every 6 months for 2 years, then once a year for 5 years, and after that every 2 years. The mean follow-up duration for this series was 10.5 years $(6$ months-21 years), corresponding to 121.6 months (6-252 months), with an average of 0.8 patients per year.

\section{Results}

Total tumor excision was possible in 16 patients, whereas total removal was not achieved in 1 patient with NF2 due to adherences within the cavernous sinus. There 


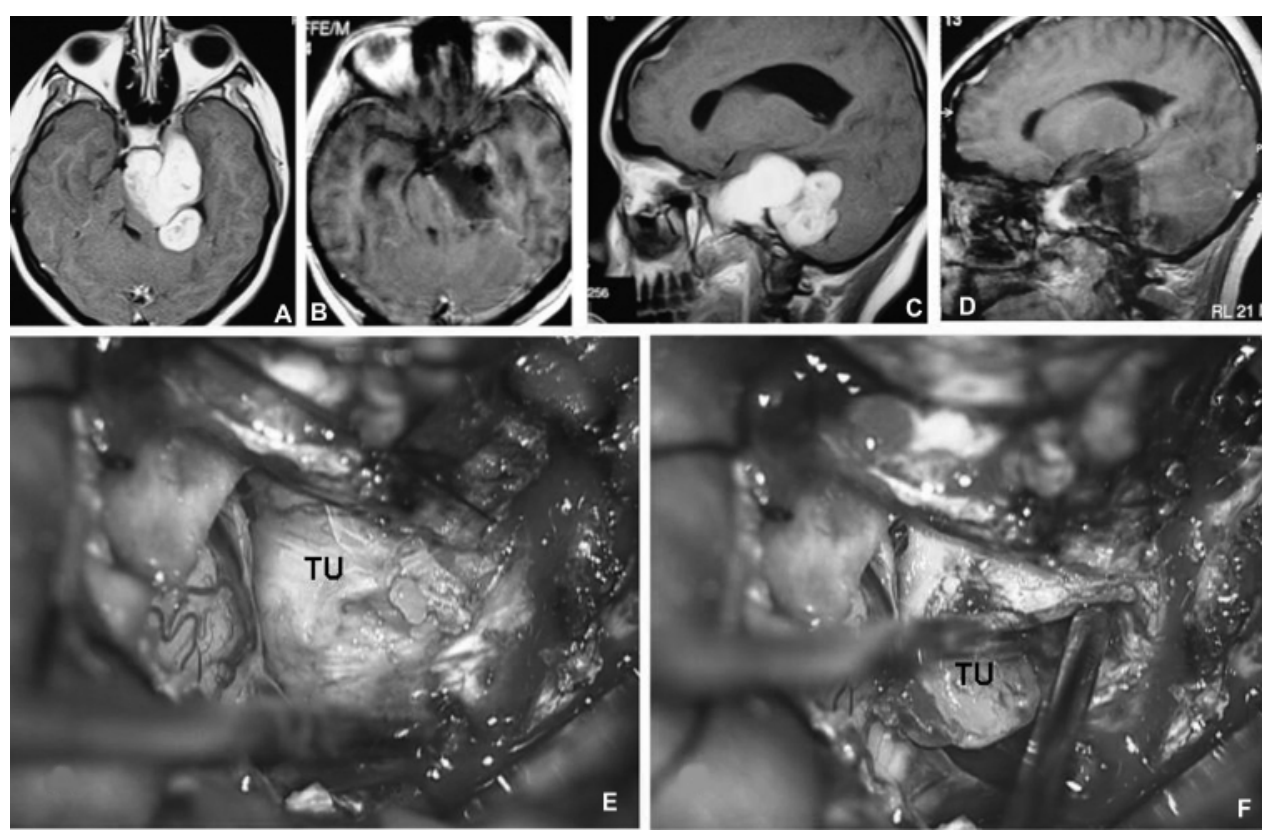

FIG. 6. Preoperative ( $A$ and $C$ ) and postoperative (B and D) MR imaging studies of a large TS (Type E) with extension within the middle and posterior fossas. E: Intraoperative photograph of the presigmoid approach for removal of the tumor. F: Intraoperative photograph showing removal of the tumor capsule from the brainstem.

were no surgery-related deaths (Table 3). Seven patients developed postoperative anesthesia in at least one branch of the trigeminal nerve. Trigeminal motor function was preserved in 7 patients. Two individuals developed a CSF leak and were successfully treated by lumbar CSF drainage. Only 1 of the 9 patients who reported facial pain preoperatively remained symptomatic postoperatively. Two patients exhibited minimal facial palsy that resolved during the follow-up period. The patient with preoperative hemiparesis showed progressive improvement after surgery. There was 1 recurrence in a patient with NF2, and that occurred 5 years after a radical removal of a Type $\mathrm{C}$ tumor. The recurrent tumor was removed, with an uneventful postoperative outcome.

\section{Discussion}

Although TSs are the second most common intracranial schwannoma, there is no typical clinical syndrome caused by TSs. Nevertheless, the most frequently noted symptoms related to trigeminal nerve dysfunction manifest as decreased sensation over the involved trigeminal nerve branch distribution, the whole face, and eventually corneal reflex alteration..$^{29,41}$ Instead of pain, sensory disturbance is mostly described as numbness and paresthesia. Some authors reported that $\sim 10 \%$ of patients will not present with trigeminal dysfunction as an initial symptom..$^{27,38}$ When present, however, facial pain can affect one or more trigeminal branches. Intensity may vary from light to lancinating pain. It may occur in paroxysms, usually without trigger zones, or may last hours. According to Day and Fukushima, ${ }^{7}$ the majority of patients with TSs have paroxysmal lancinating facial pain in episodes that tend to last longer than classic trigeminal neuralgia and do not respond to carbamazepine. Some authors $8,28,32,43$ reported that trigeminal pain may be absent with tumors originating distally in the divisions, whereas it is more common in tumors arising from the ganglion. Other reported symptoms are headaches, hearing impairment, hemifacial spasm, hemiparesis, ataxia, diplopia, signs of cerebellar involvement, increased ICP, and dysfunction of oculomotor, trochlear, and abducent nerves. ${ }^{3,27,29,32,45}$ Although rare, malignant tumors can present with involvement in all 3 branches..$^{20,22}$

Adequate preoperative neuroradiological evaluation is fundamental for establishing a correct diagnosis and treatment. Bone anatomy of the skull base is better evaluated with CT scanning and plain radiographs, which generally reveal sharp amputation of the petrous apex, anterior-medial erosion of the petrous bone, and enlargement of the foramen ovale. ${ }^{21,30,34}$ On CT scans, TSs usually appear iso- to hyperintense compared with the surrounding brain, with clearly defined margins that strongly and uniformly enhance after intravenous contrast administration. ${ }^{16,33}$ Cystic changes may also be observed. An MR

TABLE 2: Preoperative clinical symptoms in 17 patients with TSs

\begin{tabular}{lc}
\hline \multicolumn{1}{c}{ Symptom } & No. of Patients (\%) \\
\hline trigeminal hypesthesia & $9(53.0)$ \\
facial pain & $9(53.0)$ \\
headache & $6(35.3)$ \\
hearing symptoms & $3(17.6)$ \\
seizure & $3(17.6)$ \\
diplopia & $2(11.8)$ \\
ataxia & $2(11.8)$ \\
hemiparesis \& increased ICP w/ papilledema & $1(5.9)$ \\
\hline
\end{tabular}



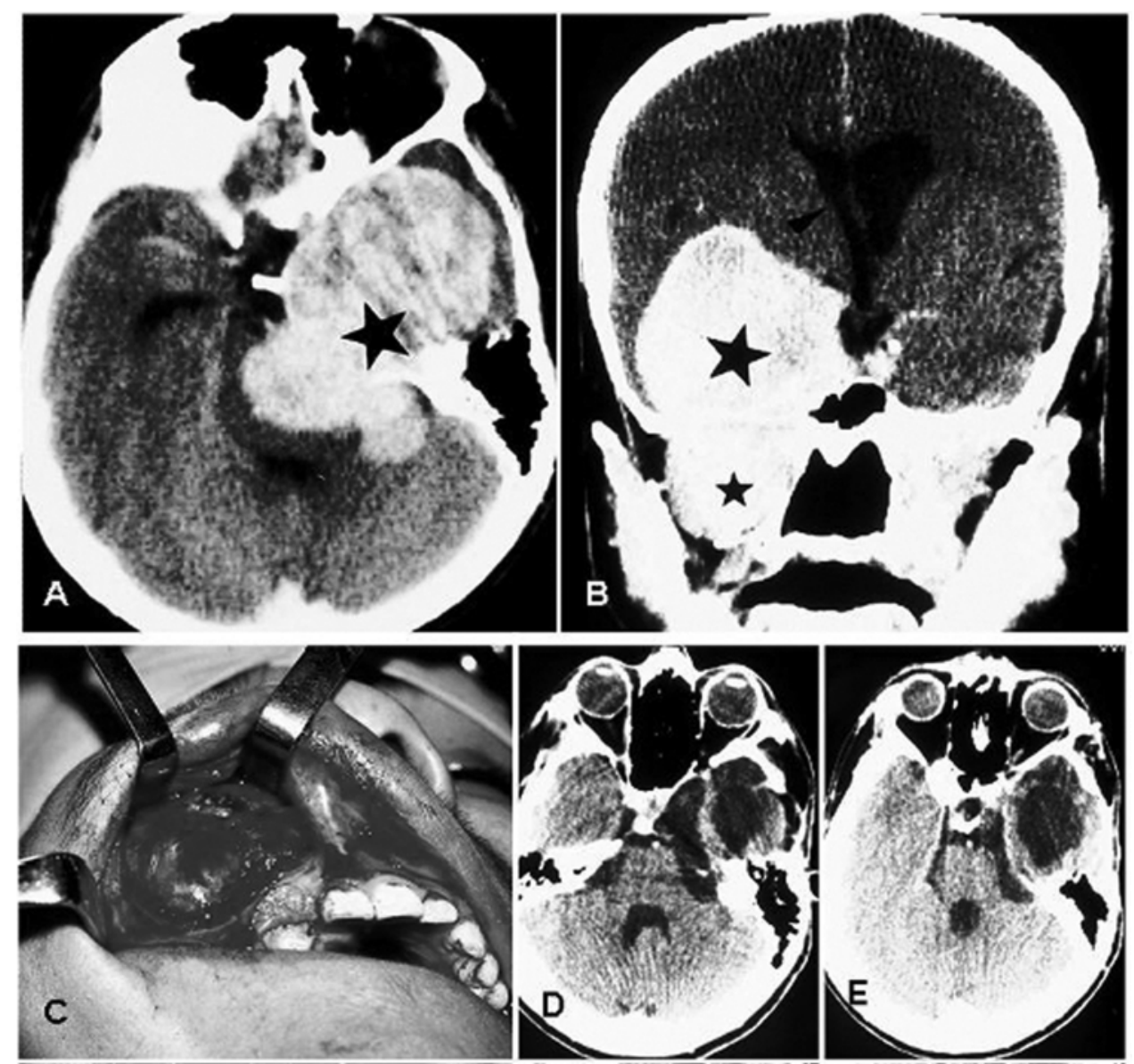

FIG. 7. Preoperative CT scans of a large TS (Type F) with extension in the posterior fossa and maxillary sinus (A and B; stars). C: Intraoperative photograph showing transmaxillary tumor exposure. D and E: Postoperative CT scans.

imaging study gives the best information concerning the localization and extent of the tumor in addition to revealing displacement of neighboring structures and involvement of the cavernous sinus and vessels. On MR imaging, TSs appear hypointense in T1-weighted and hyperintense on T2-weighted images, with marked and homogeneous enhancement after intravenous Gd administration. ${ }^{40}$ The differential diagnosis includes meningiomas, epidermoid cysts, metastasis, chondrosarcomas, chordomas, chondromas, vestibular schwannomas, and maxillary sinus tumors.

Management strategy for TSs involves clinical obser-

TABLE 3: Results of surgical procedures in 17 patients with TSs

\begin{tabular}{lc}
\hline \multicolumn{1}{c}{ Op Outcomes } & No. of Patients $(\%)$ \\
\hline total resection & $16(94.1)$ \\
near-total resection & $1(5.9)^{*}$ \\
recurrence & $1(5.9) \dagger$ \\
op-related death & 0 \\
\hline
\end{tabular}

* Tumor adhered to the cavernous sinus in a patient with NF2.

$\dagger$ A patient with NF2 had recurrence 5 years after radical tumor removal. vation followed by MR imaging follow-up for incidental tumors, surgical removal, and, alternatively, radiotherapy or radiosurgery. ${ }^{2,15,19,24,25,47}$ Complete or near-total surgical removal can be achieved in $>70 \%$ of the patients by means of skull base approaches and microsurgical dissection. Involvement of the cavernous sinus is one cause of subtotal resection. In most cases, a clear plane of cleavage between the tumor capsule and the cavernous sinus structures can be found, allowing complete dissection and total resection. In the modern neurosurgical era, recurrence of TS is rare and the outcome is usually favorable; the most frequent symptom after surgery is trigeminal hypesthesia, which is transient in most cases. Facial pain may persist after surgery, but most patients report improvement or total relief during follow-up. Diplopia, CSF leakage, meningitis, and hydrocephalus have been also described as possible complications. Most new cranial nerve deficits present resolution within 4-6 months.

Although good results have been reported with radiosurgery, this technique is reserved for small, nonresectable, and residual tumors within the cavernous sinus. Long-term follow-up of patients treated with this method is still needed to evaluate the exact role of radiosurgery in the late control of these lesions..$^{1,17-19,31,35,36,37,44}$ It is evident, however, that no patient will ever be cured of this benign tumor with radiotherapy or radiosurgery. 


\section{R. Ramina et al.}

Several contemporary series have demonstrated no deaths or major surgical complications with radical removal of TSs. ${ }^{711,15,41}$ In a classic series of 44 patients reported by Dolenc, ${ }^{11}$ total resection was achieved in $100 \%$ of the patients, including 5 who had undergone incomplete resection elsewhere and underwent another surgery to excise the remainder of the tumor. The authors recommend an epidural approach to schwannomas originating in the fifth cranial nerve peripherally to the Gasserian ganglion and either an epidural-transdural or an epiduraltransdural-transpetrous approach to lesions originating in the Gasserian ganglion or in the root of the fifth nerve.

Patients who had experienced preoperative atypical trigeminal pain reported a resolution of their symptoms after surgery. No additional treatment such as radiosurgery, other forms of irradiation, or chemotherapy was necessary.

\section{Conclusions}

Based on our experience and published data, we believe that the best treatment for TSs is complete microsurgical removal of the lesion, and that this treatment should be considered the gold standard therapeutic modality for the majority of cases when performed in selected, wellestablished, skull base neurosurgical centers.

Finally, the modifications proposed in this article for the classification of Samii et al. ${ }^{41}$ of tumor extension allows a better choice of surgical approach and anticipation of surgical difficulties.

\section{Disclaimer}

The authors report no conflict of interest concerning the materials or methods used in this study or the findings specified in this paper.

\section{Acknowledgment}

His coauthors thank Dr. Erasmo Barros da Silva Júnior for the drawings of the tumor types.

\section{References}

1. Akiyama T, Ikeda E, Kawase T, Yoshida K: Pseudocapsule formation after gamma knife radiosurgery for trigeminal neurinoma:case report. Neurol Med Chir (Tokyo) 45:526529,2005

2. Al-Mefty O, Ayoubi S, Gaber E: Trigeminal schwannomas: removal of dumbbell-shaped tumors through the expanded Meckel cave and outcomes of cranial nerve function. J Neurosurg 96:453-463, 2002

3. Arseni C, Dumitrescu L, Constantinescu A: Neurinomas of the trigeminal nerve. Surg Neurol 4:497-503, 1975

4. Bambakidis NC, Kakarla UK, Kim LJ, Nakaji P, Porter RW, Daspit CP, et al: Evolution of surgical approaches in the treatment of petroclival meningiomas: a retrospective review. Neurosurgery 62:1182-1191, 2008

5. Bordi L, Compton J, Symon L: Trigeminal neuroma. Surg Neurol 31:272-276, 1989

6. Burt AM: Textbook of Neuroanatomy: Synopsis of the Cranial Nerves. Philadelphia: WB Saunders Company, 1993, pp 403-430

7. Day JD, Fukushima T: The surgical management of trigeminal neuromas. Neurosurgery 42:233-241, 1998
8. De Benedittis G, Bernasconi V, Ettorre G: Tumors of the fifth cranial nerve. Acta Neurochir (Wien) 38:37-64, 1977

9. Dolenc VV: Anatomy and Surgery of the Cavernous Sinus. Vienna: Springer-Verlag, 1989

10. Dolenc VV: Approaches to and techniques of surgery within the cavernous sinus, in Torrens M, Al-Mefty O, Kobayashi S: Operative Skull Base Surgery. New York: Churchill Livingstone, 1997, pp 207-236.

11. Dolenc VV: Frontotemporal epidural approach to trigeminal neurinomas. Acta Neurochir (Wien) 130:55-65, 1994

12. Dolenc VV: Surgery of vascular lesions of the cavernous sinus. Clin Neurosurg 36:240-255, 1990

13. Dolenc VV, Skrap M, Sustersic J, Skrbec M, Morina A: A transcavernous-transsellar approach to the basilar tip aneurysms. Br J Neurosurg 1:251-259, 1987

14. Frazier $\mathrm{CH}$ : An operable tumor involving the gasserian ganglion. Am J Med Sci 156:483-490, 1918

15. Goel A, Muzumdar D, Raman C: Trigeminal neuroma: analysis of surgical experience with 73 cases. Neurosurgery 52:783-790, 2003

16. Goldberg R, Byrd S, Winter J: Varied appearance of trigeminal neuromas on CT. AJR Am Roentgenol 134:57-60, 1980

17. Guthikonda B, Theodosopoulos PV, van Loveren H, Tew JM $\mathrm{Jr}$, Pensak ML: Evolution in the assessment and management of trigeminal schwannoma. Laryngoscope 118:195-203, 2008

18. Hamm KD, Gross MW, Fahrig A, Surber G, Henzel M, Kleinert G, et al: Stereotactic radiotherapy for the treatment of nonacoustic schwannomas. Neurosurgery 62:29-36, 2008

19. Hasegawa T, Kida Y, Yoshimoto M, Koike J: Trigeminal schwannomas: results of Gamma Knife surgery in 37 cases. J Neurosurg 106:18-23, 2007

20. Hedeman LS, Lewinsky BS, Lochridge GK, Trevor R: Primary malignant schwannoma of the gasserian ganglion: Report of two cases. J Neurosurg 48:279-283, 1978

21. Holman CB, Olive I, Svien HJ: Roentgenologic features of neurofibromas involving the gasserian ganglion. Am J Roentgenol Radium Ther Nucl Med 86:148-153, 1961

22. Jefferson G: The trigeminal neurinomas with some remarks on malignant invasion of the gasserian ganglion. Clin Neurosurg 1:11-54, 1953

23. Knudsen V, Kolze V: Neurinoma of the gasserian ganglion and the trigeminal root. Report of four cases. Acta Neurochir (Wien) 26:159-164, 1972

24. Konovalov AN, Spallone A, Mukhamedjanov DJ, Tcherekajev VA, Makhmudov UB: Trigeminal neurinomas. A series of 111 surgical cases from a single institution. Acta Neurochir (Wien) 138:1027-1035, 1996

25. Kuo JS, Chen JCT, Yu C, Zelman V, Giannotta SL, Petrovich $\mathrm{Z}$, et al: Gamma knife radiosurgery for benign cavernous sinus tumors: quantitative analysis of treatment outcomes. Neurosurgery 54:1385-1394, 2004

26. Lang J: Anatomy of the posterior cranial fossa, in Sekhar LN, Janecka IP (eds): Surgery of Cranial Base Tumors. New York: Raven Press, 1993, pp 131-146

27. Lesoin F, Rousseaux M, Villette L, Autricque A, Dhellemmes $\mathrm{P}$, Pellerin P, et al: Neurinomas of the trigeminal nerve. Acta Neurochir (Wien) 82:118-122, 1986

28. Levinthal R, Bentson JR: Detection of small trigeminal neurinomas. J Neurosurg 45:568-575, 1976

29. McCormick PC, Bello JA, Post KD: Trigeminal schwannoma. Surgical series of 14 cases with review of the literature. J Neurosurg 69:850-860, 1988

30. Mello LR, Tänzer A: Some aspects of trigeminal neurinomas. Neuroradiology 4:215-221, 1972

31. Miller JP, Acar F, Burchiel KJ: Trigeminal neuralgia and vascular compression in patients with trigeminal schwannomas: case report. Neurosurgery 62:974-975, 2008 
32. Nager GT: Neurinomas of the trigeminal nerve. Am J Otolaryngol 5:301-331, 1984

33. Naidich TP, Lin JP, Leeds NE, Kricheff II, George AE, Chase NE et al: Computed tomography in the diagnosis of extra-axial posterior fossa masses. Radiology 120:333-339, 1976

34. Palacios E, MacGee EE: The radiographic diagnosis of trigeminal neurinomas. J Neurosurg 36:153-156, 1972

35. Pan L, Wang EM, Zhang N, Zhou LF, Wang BJ, Dong YF, et al: Long-term results of Leksell gamma knife surgery for trigeminal schwannomas. J Neurosurg 102:220-224, 2005

36. Peker S, Bayrakli F, Kiliç T, Pamir MN: Gamma-knife radiosurgery in the treatment of trigeminal schwannomas. Acta Neurochir (Wien) 149:1133-1137, 2007

37. Phi JH, Paek SH, Chung HT, Jeong SS, Park CK, Jung HW, et al: Gamma Knife surgery and trigeminal schwannoma: is it possible to preserve cranial nerve function? J Neurosurg 107:727-732, 2007

38. Pollack IF, Sekhar LN, Jannetta PJ, Janecka IP: Neurilemmomas of the trigeminal nerve. J Neurosurg 70:737-745, 1989

39. Ramina R, Maniglia JJ, Paschoal JR, Fernandes YB, Neto $\mathrm{MC}$, Honorato DC: Reconstruction of the cranial base in surgery for jugular foramen tumors. Neurosurgery 56:337-343, 2005

40. Rigamonti D, Spetzler RF, Shetter A, Drayer BP: Magnetic resonance imaging and trigeminal schwannomas. Surg Neurol 28:67-70, 1987

41. Samii M, Migliori MM, Tatagiba M, Babu R: Surgical treatment of trigeminal schwannomas. J Neurosurg 82:711-718, 1995

42. San Millán Ruiz D, Gailloud P, de Miquel Miquel MA, Muster M, Dolenc VV, Rufenacht DA, et al: Laterocavernous sinus. Anat Rec 254:7-12, 1999

43. Schisano G, Olivecrona $\mathrm{H}$ : Neurinomas of the gasserian ganglion and trigeminal root. J Neurosurg 17:306-322, 1960

44. Sheehan J, Yen CP, Arkha Y, Schlesinger D, Steiner L: Gamma knife surgery for trigeminal schwannoma. J Neurosurg 106:839-845, 2007

45. Shrivastava RK, Sem C, Post KD: Trigeminal schwannomas, in Winn HR (ed): Youmans Neurological Surgery. Philadelphia: WB Saunders Company, 2004 pp 1343-1350

46. Yasui T, Hakuba A, Kim SH, Nishimura S: Trigeminal neurinomas: operative approach in eight cases. J Neurosurg 71:506-511, 1989

47. Yoshida K, Kawase T: Trigeminal neurinomas extending into multiple fossae: surgical methods and review of the literature. J Neurosurg 91:202-211, 1999

Manuscript submitted June 9, 2008.

Accepted October 10, 2008.

Address correspondence to: Marília Grando Sória, Rua Jeremias Maciel Perretto 300, Postal code 81210-310, Curitiba, Paraná, Brazil.email: mgsoria@gmail.com.

\section{Comment by Dr. Vinko Dolenc}

Prior to the parasellar microanatomy studies and understanding of this tumor's complexity, treatment of TSs has been a difficult task. It is true that the diagnosis of these tumors was also very difficult prior to CT and MR imaging. Symptoms and signs in even large trigeminal neurinomas might be and usually are minimal. In a considerable number of cases, the diagnosis of trigeminal neurinoma is an incidental finding on imaging performed for other reasons. The anatomical considerations are extremely important: Gasserian ganglion and V3 are exclusively outside of the parasellar space. Even V1 and V2 are not in the parasellar space but in its lateral wall, "encased" in delicate membranes from both sides. The roots of the fifth cranial nerve-its compact and its plexiform segments-are in the subarachnoid space as it is the smaller or larger part of the Gasserian ganglion.

On the basis of these anatomical data, it is clear that the surgical approaches should be tailored accordingly. With the extradural approach, most trigeminal neurinomas arising from V1, V2, V3, and peripheral region(s) of the Gasserian ganglion may be completely exenterated. When the lesion is located centrally from the Gasserian ganglion or in continuation from the middle to the posterior cranial fossa, then the extradural-intradural-transpetrous approach is the most preferred one. With this approach, all the tumors originating from the segment of the root of the fifth cranial nerve as well as from the Gasserian ganglion itself can be excised completely. Only exceptionally, the retrosigmoid approach might be chosen, and this is in cases in which the preoperative diagnosis is not $100 \%$ sure for trigeminal neurinoma.

For extracranial trigeminal neurinomas, splanchnocranial direct approaches might be practical, although when the tumor is also in the middle cranial fossa, it is the best way at least to combine the epidural transcranial approach with the splanchnocranial approach to achieve complete extirpation of the lesion. The 2 remaining problems associated with neurinomas of the region are as follows: 1) neurinomas as a part of neurofibromatosis, and 2) neurinomas originating in the parasellar space (cavernous sinus) from the sympathetic fibers accompanying the internal carotid artery. In these 2 entities, a radical excision is difficult-if possible at all-and most probably the best way is to combine the surgical treatment with radiosurgery.

In our practice, the presigmoid approach has never been used, because the necessity for it never existed. Regardless of the size of the lesion, the intradural approach as it is described in this paper is not necessary for the extradural approach to be executed correctly. The orbitozygomatic approach is also not necessary for this kind of lesion as well as splitting of the sylvian fissure. Surgery for trigeminal neurinomas is in fact the safest surgery for the region, and postoperative deficits should not exceed the preoperative ones; on the contrary, it should be less. There is one thing worth mentioning, and that is severe trigeminal pain, which may initiate the diagnosis of the disease; and when this occurs, it is the result of bleeding into the tumorous lesion, which suddenly increases the pressure against the surrounding structures. Such pain does subside in about a 1-month interval if the patient does not accept surgical treatment before. And what is interesting is that diplopia, which might be the first symptom (that is, it may occur earlier than sensory deficits), is usually (in $>50 \%$ of cases) due to displacement of the sixth cranial nerve and its being pushed against the hard and sharp edge of the bone inferolaterally. This cause of diplopia is much more frequent than diplopia due to irritation of the third and fourth cranial nerves. 